Annales Geophysicae (2001) 19: 1273-1288 C European Geophysical Society 2001

\title{
The Electron Drift Instrument on Cluster: overview of first results
}

\author{
G. Paschmann ${ }^{1}$, J. M. Quinn 2 , R. B. Torbert ${ }^{2}$, H. Vaith ${ }^{1}$, C. E. McIlwain ${ }^{3}$, G. Haerendel ${ }^{4}$, O. H. Bauer ${ }^{1}$, T. Bauer ${ }^{1}$, \\ W. Baumjohann ${ }^{5}$, W. Fillius ${ }^{3}$, M. Förster ${ }^{1}$, S. Frey ${ }^{1}$, E. Georgescu ${ }^{1}$, S. S. Kerr ${ }^{3}$, C. A. Kletzing ${ }^{6}$, H. Matsui ${ }^{2}$, \\ P. Puhl-Quinn ${ }^{1}$, and E. C. Whipple ${ }^{7}$ \\ ${ }^{1}$ Max-Planck-Institut für extraterrestrische Physik, 85748 Garching, Germany \\ ${ }^{2}$ University of New Hampshire, Durham, NH 03824, USA \\ ${ }^{3}$ University of California at San Diego, La Jolla, CA 92093, USA \\ ${ }^{4}$ International University Bremen, 28725 Bremen, Germany \\ ${ }^{5}$ Space Research Institute, 8042 Graz, Austria \\ ${ }^{6}$ University of Iowa, Iowa City, IA 52242, USA \\ ${ }^{7}$ University of Washington, Seattle, WA 98195, USA
}

Received: 18 April 2001 - Revised: 9 July 2001 - Accepted: 11 July 2001

\begin{abstract}
EDI measures the drift velocity of artificially injected electron beams. From this drift velocity, the perpendicular electric field and the local magnetic field gradients can be deduced when employing different electron energies. The technique requires the injection of two electron beams at right angles to the magnetic field and the search for those directions within the plane that return the beams to their associated detectors after one or more gyrations. The drift velocity is then derived from the directions of the two beams and/or from the difference in their times-of-flight, measured via amplitude-modulation and coding of the emitted electron beams and correlation with the signal from the returning electrons. After careful adjustment of the control parameters, the beam recognition algorithms, and the onboard magnetometer calibrations during the commissioning phase, EDI is providing excellent data over a wide range of conditions. In this paper, we present first results in a variety of regions ranging from the polar cap, across the magnetopause, and well into the magnetosheath.
\end{abstract}

Key words. Electron drift velocity (electric fields; plasma convection; instruments and techniques)

\section{Introduction}

The Electron Drift Instrument (EDI) measures the drift velocity of artificially injected electron beams. From this drift velocity which, by definition, is directed perpendicular to the magnetic field, the perpendicular electric field can be deduced. EDI complements the double-probe technique employed by the EFW instrument on Cluster (Gustafsson et al.,

Correspondence to: G. Paschmann (gep@mpe.de)
1997) in that the EDI measurements are essentially unaffected by the spacecraft environment and include the component of the field along the spacecraft spin axis. The present paper provides an overview of first results obtained with EDI. After a brief description of the technique and its implementation in Sect. 2 and 3, the in-flight performance and analysis methods are discussed in Sect. 4, before results, predominantly from the dayside, are presented in Sect. 5. Results from the night sector are described in a companion paper (Quinn et al., 2001, this issue).

\section{Principle of operation}

The basis of the electron-drift technique is the injection of weak beams of electrons and their detection after one or more gyrations in the ambient magnetic field. A detailed description of the technique may be found in earlier publications (Paschmann et al., 1997, 1998). Some limited in-flight experience with EDI was already obtained on the Equator-S mission (Paschmann et al., 1999; Quinn et al., 1999). Briefly, in the presence of a drift velocity $\boldsymbol{V}_{d}$, induced by an electric field $\boldsymbol{E}_{\perp}$ or a magnetic-field gradient $\nabla B_{\perp}$, the circular electron orbits are distorted into cycloids. Their shape depends on whether the beam is injected with a component parallel or anti-parallel to the drift velocity. To be able to recognize both types of orbits simultaneously, EDI uses two guns and two detectors. Figure 1 shows examples of these two orbits in the plane perpendicular to $\boldsymbol{B}$, which we refer to as the $\boldsymbol{B}_{\perp}$-plane. For each gun, only one orbit-solution exists that connects it to the detector on the opposite side of the spacecraft. Knowing the positions of the guns and the firing directions that cause the beams to hit their detectors uniquely determines 


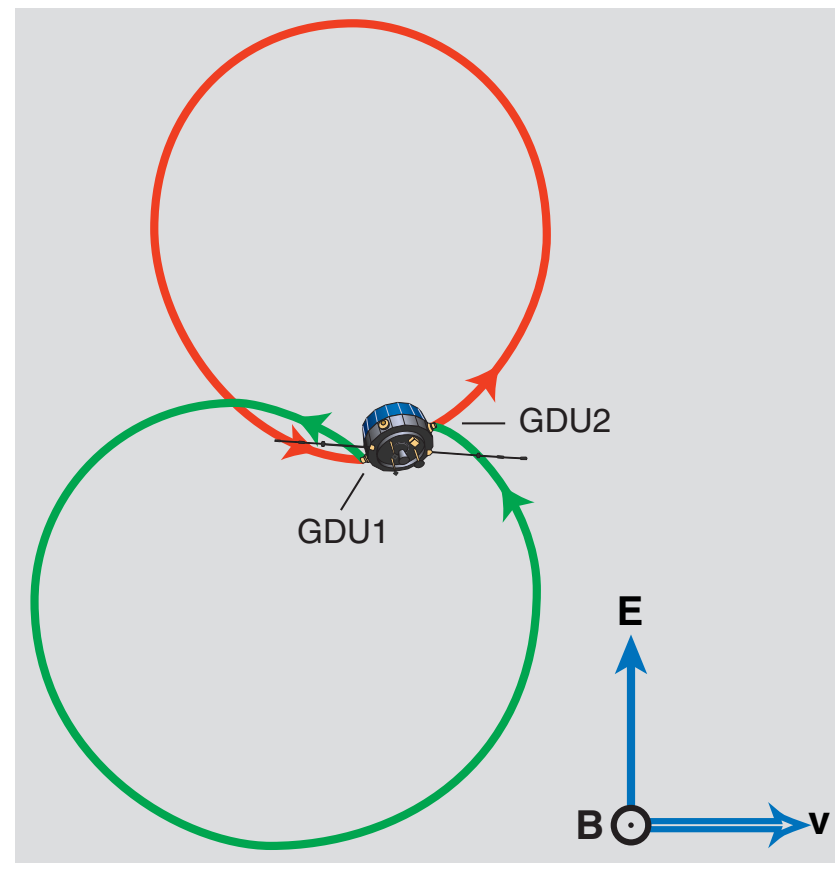

Fig. 1. EDI principle of operation. For any combination of magnetic field $\boldsymbol{B}$ and drift velocity $\boldsymbol{V}_{d}$ (assumed here to be induced by an electric field $\boldsymbol{E}$ ), only a single electron-trajectory exists that connects each gun with the detector on the opposite side of the spacecraft. The two trajectories have different path lengths and thus different times-of-flight. Note that for clarity the electron orbits are drawn for a very high drift velocity, $\boldsymbol{V}_{d}=1000 \mathrm{~km} \mathrm{~s}^{-1}$ and an unrealistically large magnetic field, $\boldsymbol{B}=12 \mu \mathrm{T}$, implying an equally unrealistically large electric field of $12 \mathrm{~V} \mathrm{~m}^{-1}$, but a reasonable drift step of $\boldsymbol{d}=3 \mathrm{~m}$. For realistic magnetic fields, the gyro radius is much larger, e.g. $1065 \mathrm{~m}$ for a $100 \mathrm{nT}$ field.

the drift velocity. This is the basis of the triangulation technique, where one directly determines the "drift-step" vector $\boldsymbol{d}$, which is the displacement of the electrons after a gyro time $T_{g}$ :

$\boldsymbol{d}=\boldsymbol{V}_{d} T_{g}$

The location in the $\boldsymbol{B}_{\perp}$-plane, from which electrons reach the detector after one gyration, can be viewed as the "target" for the electron beams, as discussed in Quinn et al. (2001, this issue).

Note that for time-stationary conditions one gun-detector pair would suffice, because the satellite spin would rotate the gun into all positions sequentially. This is exactly what was done with the Electron Beam Experiment on Geos-2 (Melzner et al., 1978), which served as the proof-of-principle for the electron-drift technique In addition to being limited to spin-period resolution, the Geos instrument had a further limitation in that it could be operated only for small $\left(<18^{\circ}\right)$ angles between the ambient magnetic field and the spacecraft spin axis. EDI, with its fully steerable beams, can follow the target continuously regardless of magnetic field orientation.

As is evident from Fig. 1, the two orbits differ in their length, and thus in the electron travel times. The electrons

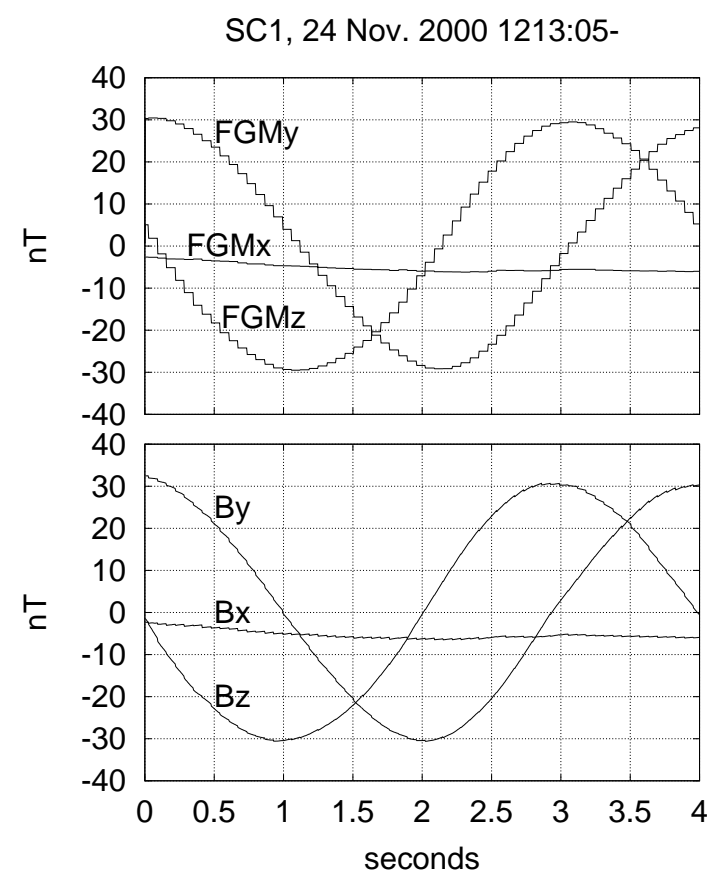

Fig. 2. Synthesis of the FGM and STAFF magnetometer data that EDI receives on-board over the Inter-Experiment Link (IEL). The top panel shows the raw FGM data, received at 16 samples/s, which appears as a stair-case because it is sampled here every $4 \mathrm{~ms}$. The bottom panel shows the combined (and rotated) data, which illustrates the success of the synthesis method explained in the text. The plot uses the spacecraft convention with $X$ along the spin axis. The sinusoidal variation in the $Y$ - and $Z$-components is due to the spacecraft spin. The plot was made from data stored in EDI's scratchRAM and dumped with the special BM3 telemetry mode that Cluster provides.

emitted with their velocity directed with a component parallel to $\boldsymbol{V}_{d}$, i.e. away from the target, have a time of flight that is shorter than $T_{g}$, while the electrons emitted towards the target have a time of flight that is longer than $T_{g}$ :

$T_{1,2}=T_{g}\left(1 \pm V_{d} / V_{e}\right)$,

where $V_{e}$ is the electron velocity. From Eq. (2) it follows immediately that the difference between the two times-offlight provides a measure of the drift velocity, $V_{d}$ :

$\Delta T=T_{1}-T_{2}=2\left(V_{d} / V_{e}\right) T_{g}=2\left(d / V_{e}\right)$,

while their sum is twice the gyro time:

$T_{1}+T_{2}=2 T_{g}$.

Noting that $T_{g}=2 \pi m_{e} / e B$, this means that the time-offlight measurements allow $B$ to be determined as well.

Drift velocities encountered on a Cluster orbit typically range from a few $\mathrm{km} \mathrm{s}^{-1}$ to less than $1000 \mathrm{~km} \mathrm{~s}^{-1}$, while the velocity of $1 \mathrm{keV}$ electrons is $18728 \mathrm{~km} \mathrm{~s}^{-1}$. According to Eq. (3), this implies that $\Delta T$ is only a small fraction of $T_{g}$, i.e. the drift introduces only a small variation in the two orbits and the associated times-of-flight. To make the 


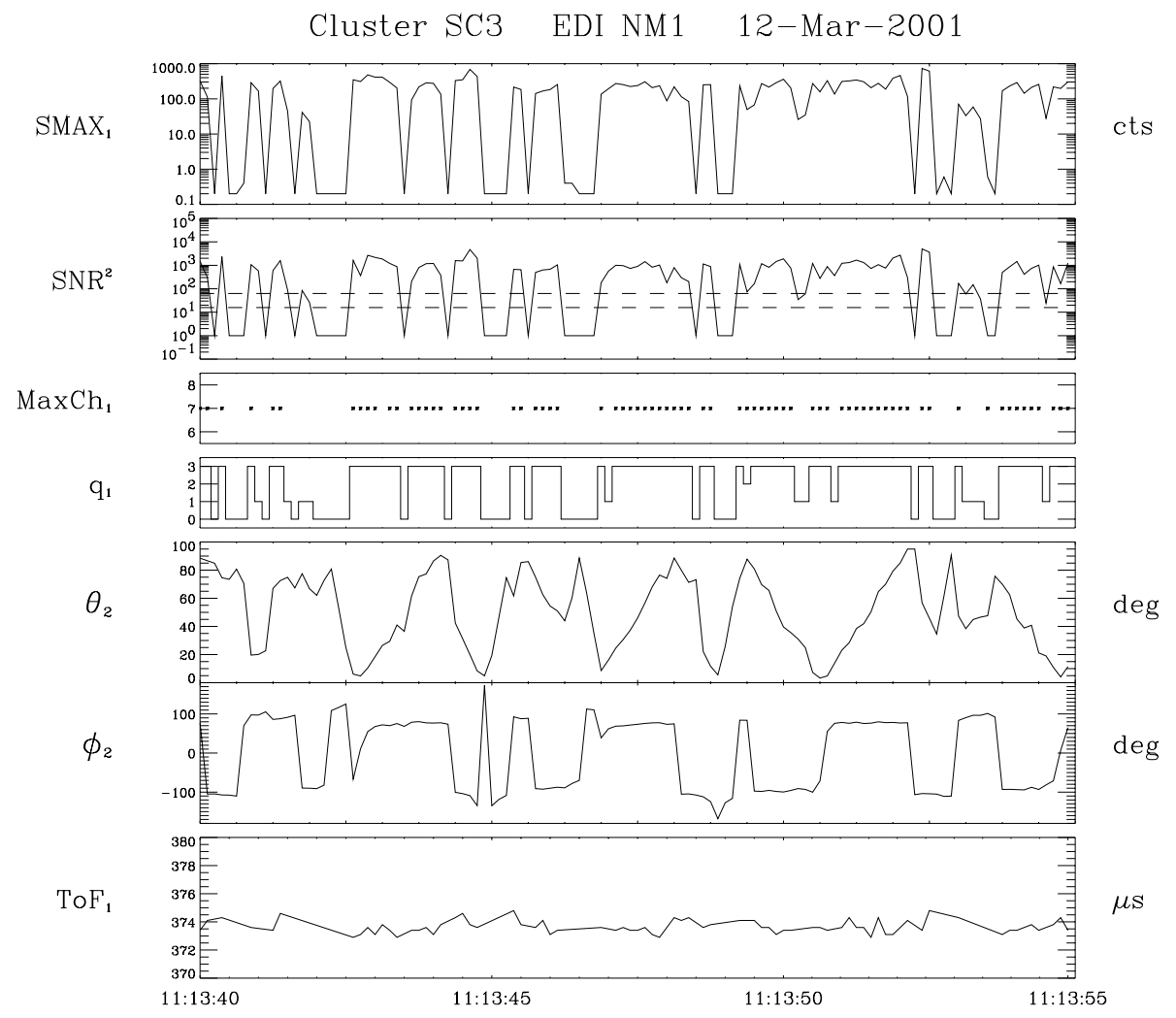

Fig. 3. EDI raw-data from Gun 2 and Detector 1 on SC 3 for a 15 -second period on 12 March 2001, when the magnetic field strength was $100 \mathrm{nT}$. The top panel shows $\mathrm{SMAX}_{1}$, the maximum counts recorded (in $2 \mathrm{~ms}$ ) in any of the 15 correlators in the Detector; the next panel shows the square of the signal-to-noise ratio, $\mathrm{SNR}^{2}$, computed from the counts in the matched and unmatched correlators; the horizontal dashed lines in this panel indicate the thresholds for $\mathrm{SNR}^{2}$ used by the on-board software to identify the beam (angle-track); the third panel shows $\mathrm{MaxCh}_{1}$, the correlator channel that received the maximum counts; when $\mathrm{MaxCh}_{1}=7$, time-track has been achieved; $q_{1}$ (fourth panel) is a quality-status indicator explained in the text; the next two panels show $\Theta_{2}$ and $\Phi_{2}$, the elevation and azimuth angles of the Gun 2 firing directions; $\mathrm{ToF}_{1}$ (last panel) is the time-of-flight of the electrons from Gun 2 to Detector 1.

difference visible, Fig. 1 is drawn for unrealistically large magnetic and electric fields. The idea to use the difference in electron times-of-flight for drift velocity or electric field measurements is due to Tsuruda et al. (1985) and was first applied by the "boomerang" instrument on Geotail (Tsuruda et al., 1998). That instrument was, however, limited to one measurement per spin, and could not accommodate all magnetic field orientations. EDI is the first instrument to combine the continuous triangulation and time-of-flight techniques, and, as already mentioned, can be operated for arbitrary magnetic field orientations. EDI was first flown on the Equator-S mission and valuable information concerning operations and on-board software was gained, although limited by the short duration of the mission.

The triangulation and time-of-flight techniques complement each other ideally. While triangulation naturally becomes increasingly inaccurate if the target moves further and further away, the time-of-flight technique becomes more accurate because, according to Eq. (3), $\Delta T$ increases with increasing drift steps, and thus becomes easier to measure. A first comparison of the two techniques on Equator-S was reported by Paschmann et al. (1999).
The electric field and gradients in the magnetic field both contribute to the drift velocity:

$V_{d}=V_{E}+V_{\nabla B}$,

where, with $W$ as the electron energy, the two drift velocities are defined as:

$$
\boldsymbol{V}_{E}=(\boldsymbol{E} \times \boldsymbol{B}) / B^{2}, \quad \boldsymbol{V}_{\nabla B}=(W / e)(\boldsymbol{B} \times \nabla B) / B^{3} .
$$

To separate $\mathbf{V}_{E}$ and $\mathbf{V}_{\nabla B}$, two electron energies are employed. For $W_{2}=2 W_{1}$ one gets:

$\boldsymbol{V}_{E}=2 \boldsymbol{V}_{1}-\boldsymbol{V}_{2}, \quad \boldsymbol{V}_{\nabla B}\left(W_{1}\right)=\boldsymbol{V}_{2}-\boldsymbol{V}_{1}$,

where $\boldsymbol{V}_{1}$ and $\boldsymbol{V}_{2}$ refer to the (total) drift velocities measured at $W_{1}$ and $W_{2}$, respectively.

So far we have tacitly assumed that the beam electrons are detected after a single gyration. Electrons that have gyrated $N$ times will have a drift step and $\Delta T$ that are $N$ times larger. As we will see, electrons having gyrated several times ("multirunners") are indeed observed. We will refer to $N$ as the multirunner order. 


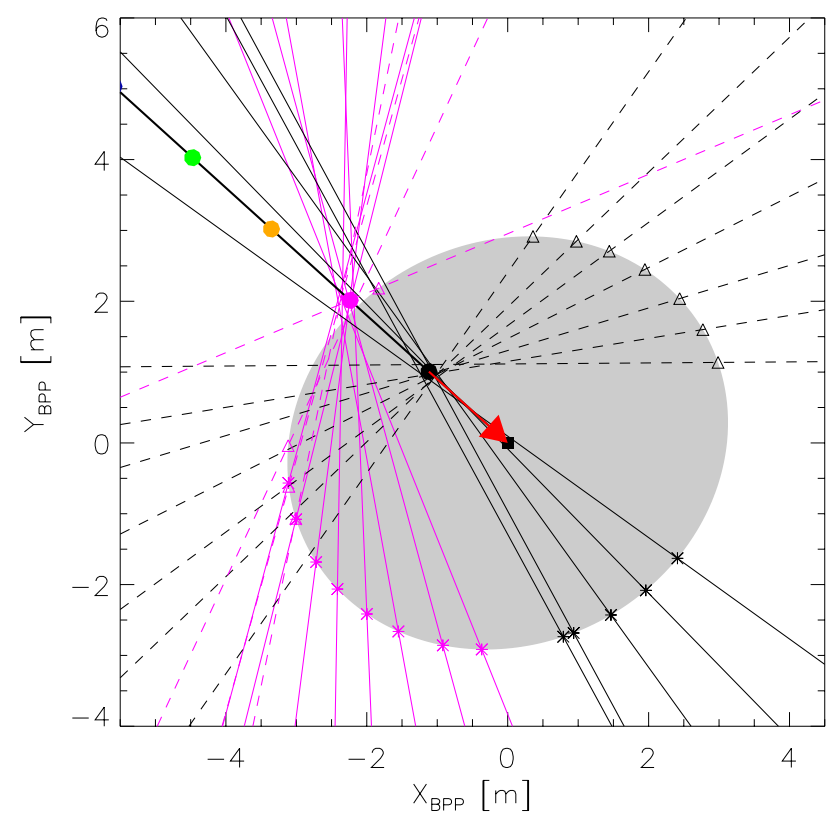

Fig. 4. Example of the triangulation analysis. The figure shows the spacecraft, the guns and the beam firing directions, all projected into the $\boldsymbol{B}_{\perp}$-plane, for a $4 \mathrm{~s}$ interval on 5 March 2001 (see Fig. 12). Gun 1 and Gun 2 locations are indicated by asterisks and triangles, respectively, the beams emanating from these guns are shown as dashed and solid lines, respectively. The solid circles are placed at integer multiples of the drift step from the center of the spacecraft, and are obtained from the best fit to all the beams in this interval. The (red) vector from the solid black circle to the center of the spacecraft is the drift step. The correct identification of the magenta-colored beams as double runners obviously has a profound effect on the drift step, identified by the black circle, which is the target for the single runners (black beams). No higher-order runners are present in this case. The drift step is $1.5 \mathrm{~m}$ in this case.

\section{Implementation}

\subsection{Gun-detector characteristics}

EDI consists of two gun-detector units (GDUs) and a controller unit. The GDUs are mounted on opposite sides of the spacecraft and have oppositely directed fields of view. The guns are capable of firing in any direction within more than a hemisphere $\left(0-96^{\circ}\right.$ polar angle) to accommodate arbitrary magnetic and electric field directions. Similarly, the detectors can detect beams coming from any selectable direction within more than a hemisphere $\left(0-100^{\circ}\right.$ polar angle).

Beams have an angular width of approximately $1^{\circ}$ at small polar emission angles, increasing to 4 -by- $1^{\circ}$ at large polar angles. Electron energies can be switched between $0.5 \mathrm{keV}$ and $1.0 \mathrm{keV}$. Separate calibration tables for the two energies are used to convert beam firing directions into the corresponding deflection voltages.

The flux-density of the returning electrons is proportional to $I_{b} B^{3} / E$ (except when the drift step is small). To accommodate the large variations in $B$ and $E$ along the Clus- ter orbit, the beam currents, $I_{b}$, can be changed over more than two orders of magnitude (from $1 \mathrm{nA}$ to several hundred $\mathrm{nA}$ ). Beam currents are initialized based on the ambient magnetic field strength and then varied automatically based on the tracking success.

Similarly, by using different combinations of highvoltages for the detector optics, a large variety of effective aperture areas, $A$, and geometric factors, $G$, can be realized. $A$ and $G$ determine the sensitivity to beam and background electrons, respectively. By choosing the right combination of $G$ and $A$, adequate signal and signal-to-noise-ratio (SNR) levels can be maintained over a wide range of field strengths and background electron fluxes. Tables of the optics voltages that achieve specific combinations of $G$ and $A$ are referred to as "Optics States". The automatic Optics-State navigation is based on measured flux levels and magnetic field strength.

\subsection{Time-of-flight measurements}

In order to measure the electron times-of-flight, as well as to distinguish beam electrons from the background of ambient ectrons, the electron beams are amplitude-modulated with a pseudo-noise (PN) code. Nakamura et al. (1989) were the first to use a PN-code for (ion) drift measurements.

The EDI time-of-flight system has been described in earlier publications (Vaith et al., 1998; Paschmann et al., 1998, 1999). Briefly, a set of 15 correlators analyzes the phasing of the detector counts relative to the beam code. Before beam acquisition has been achieved, all correlators will show the same counts (to within Poisson statistics) from the ambient electron background. Once the beam is acquired ("angle-track"), the correlator whose delay matches the electron flight-time will have the maximum number of counts. A delay-lock-loop continuously shifts the code-phases of the correlators to keep the maximum centred in a specific channel ("time-track"). By keeping track of the net change in code-phase, one obtains a measure of the changes in time-offlight.

Commensurate with the number of correlators, EDI employs primarily a 15-chip code. This way the signal is recorded in one of the correlators regardless of the actual time-of-flight. But because the accuracy is related to the chip-length, $T_{\text {chip }}$, the code-duration is kept short, much shorter than $T_{g}$. The electron time-of-flight is therefore equal to an integer number of code-lengths plus a fraction, of which only the fraction is measured by the correlators directly. However, by choosing a code-length equal to $T_{g} / 5$ or $T_{g} / 10$, where $T_{g}$ is estimated from the on-board FGM data, the number of complete wrap-arounds of the code can be recovered unambiguously. To track small time-of-flight variations, the code is shifted with a resolution of typically $T_{\text {chip }} / 32$. Simulations of the correlator performance indicate that the accuracy of individual time-of-flight measurements is about $T_{\text {chip }} / 8$. To account for the large variations in $T_{g}$ along the Cluster orbit, the code-length can be varied between approximately $15 \mu \mathrm{s}$ and $2 \mathrm{~ms}$. A problem with the short code is that it does not discriminate against multi-runners. Regardless of 


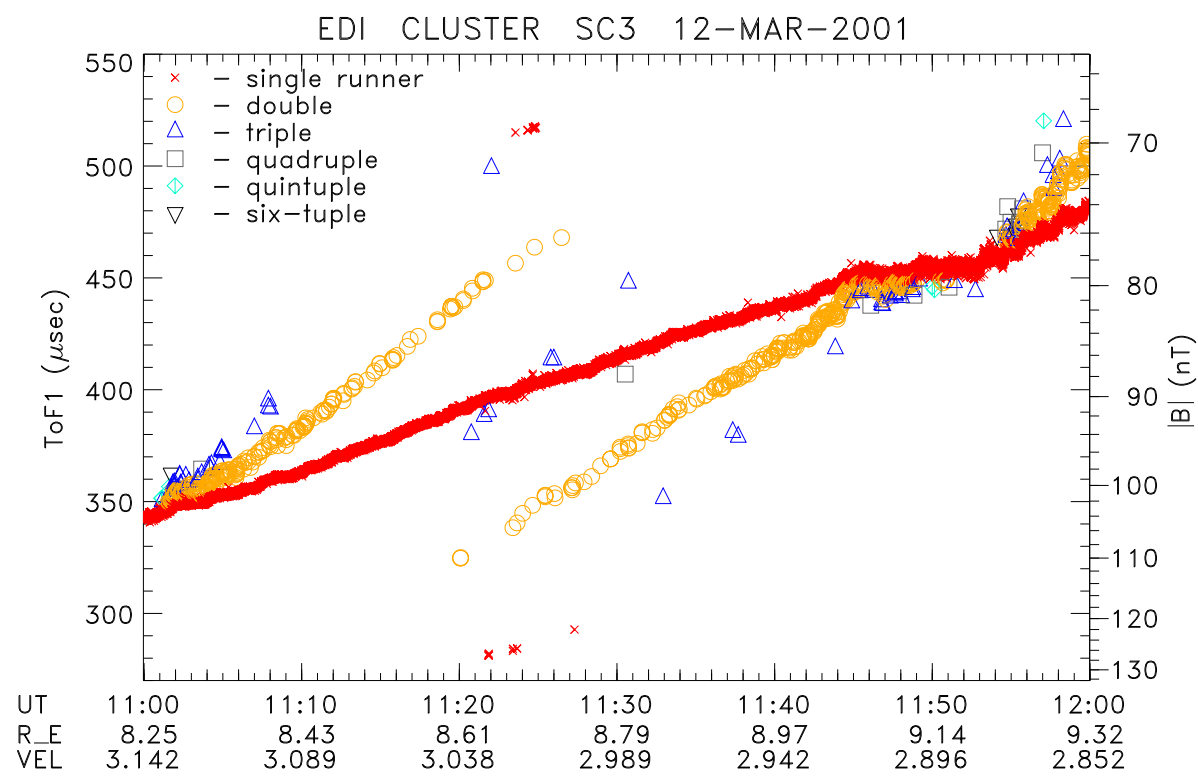

Fig. 5. Example of measured times-of-flight for Detector 1 . The order of the multi-runners, identified by different symbols, has been determined by the method explained in the text. The scale on the right shows the magnetic field strength computed from the times-of-flight.

how many times the electrons have gyrated before hitting the detector, the signal will appear in one of the 15 correlators. We therefore have introduced a second, much longer code. It has 127 chips, and its length can exceed $4 T_{g}$. By placing the 15 correlators at a time-delay near $T_{g}$, only single-runners are detected (unless runners of order 5 or higher are present as well). As the increased chip-length implies lower accuracy in time-of-flight measurements, the long code is only used in strong $(>100 \mathrm{nT}$ ) fields where multi-runners most frequently occur.

\subsection{Beam acquisition and tracking}

To find the beam directions that will hit the detector, EDI sweeps each beam in the plane perpendicular to $\boldsymbol{B}$ at a fixed angular rate (typically $0.2^{\circ} / \mathrm{ms}$ ) until a signal has been acquired by the detector. Once signal has been acquired, the beams are swept back and forth to stay on target. Beam detection is not determined from the changes in the countrates directly, but from the square of the beam counts divided by the background counts from ambient electrons, i.e. from the square of the instantaneous signal-to-noise-ratio, $S N R^{2}$. This quantity is computed from the counts recorded simultaneously in the matched and unmatched correlator channels. If it exceeds a threshold, this is taken as evidence that the beam is returning to the detector. The thresholds for $S N R^{2}$ are chosen dependent on background fluxes, and vary between 35 and 200. These values have been selected after extended experimentation during commissioning, and represent a compromise between getting false hits (induced by strong variations in background electron fluxes) and missing true beam hits. The basic software loop that controls EDI operations is executed every $4 \mathrm{~ms}$. As the times when the beams hit their detectors are neither synchronized with the telemetry nor equidistant, EDI does not have a fixed time-resolution.

\subsection{On-board magnetic field data handling}

EDI searches for the drift-step target in the plane perpendicular to $\boldsymbol{B}$, and therefore needs information on the local instantaneous field as frequently as possible. Flux-gate magnetometer data are available on board over the interexperiment-link (IEL) with the FGM instrument (Balogh et al., 1997). These data must first be time-tagged, because FGM sampling is not synchronized to the spacecraft clock, and then corrected for calibration angles, sensitivities, and offsets, and finally rotated by $6.5^{\circ}$ to the spacecraft body axes. As the FGM data are available over the IEL only 16 times per second, the EDI controller constructs the field at higher frequencies using the analog signals from the three axes of the search-coil data provided by the STAFF instrument also over the IEL (Cornilleau-Wehrlin et al., 1997). To first order, the search coil signal is integrated and added periodically to the FGM values, after rotations that account for the different coordinate systems of the two magnetometers. However, the frequency response of STAFF, as seen in Fig. 4 of Cornilleau-Wehrlin et al. (1997), differs from a pure differentiator in two respects. First, there is a high frequency roll-off above $40 \mathrm{~Hz}$. EDI accepts this frequency basically as the limit at which it can track $\boldsymbol{B}$. Second, there is a lowfrequency cut-off that is inherent in the coil-pickup response. This reduces the signal primarily at the spacecraft spin frequency and is compensated by adding the properly phaseadjusted component at that frequency. Figure 2 shows the reconstructed signal for a time interval of about one spin period. The success of the reconstruction can be measured by the extent to which the discontinuities seen at the FGM update rate have been reduced. 


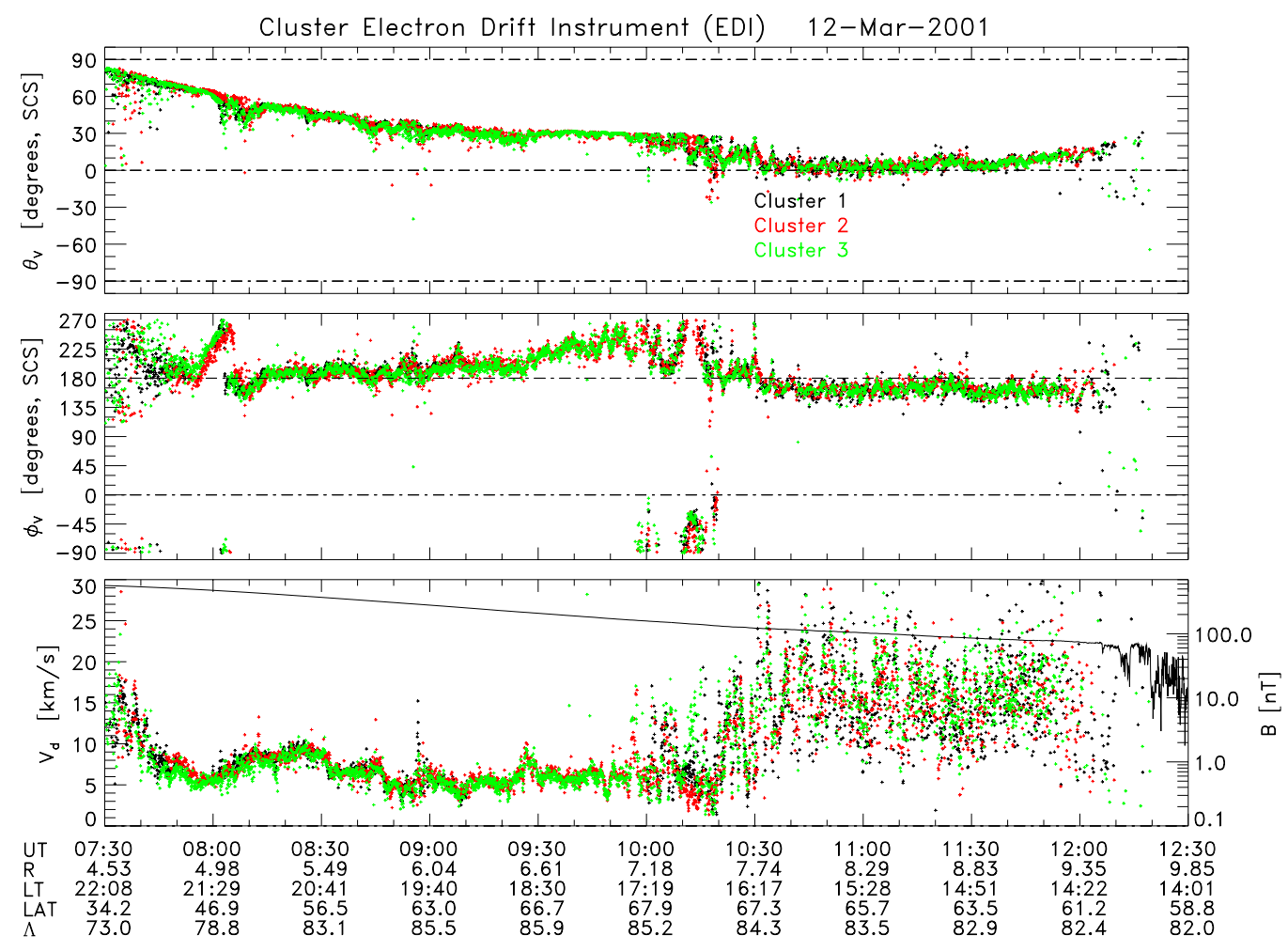

Fig. 6. EDI spin-resolution data from three spacecraft for the outbound pass on 12 March 2001, 07:30-12:30 UT. The top two panels show the elevation and azimuth angles of the drift velocity, $\Theta_{v}$ and $\Phi_{v}$, in SCS coordinates. Because the flow is towards $180^{\circ}$ much of the time, the scale for the azimuth is shown from $-90^{\circ}$ to $270^{\circ}$. The third panel shows the magnitude of the drift velocity, and, as a black line, the magnetic field magnitude measured by FGM on SC 1 for reference. The spacecraft position (in GSE) and invariant latitude, given along the bottom, are for SC 1. The data are plotted on the same scale for the three spacecraft and are placed at spin-center times, which are not identical because the spins of the Cluster spacecraft are not synchronized. As the green symbols (for SC 3) are plotted last, they are the only ones visible in regions of close agreement.

An accuracy of better than $0.5^{\circ}$ in the direction of $\boldsymbol{B}$ is required because the width of the beam is about $1^{\circ}$. Naturally, this poses stringent requirements on the calibration of the magnetometer data, as reconstructed by EDI from both the FGM and STAFF information, as described above. Errors of order $1 \mathrm{nT}$ are of no concern to EDI if the total field is sufficiently large. However, for fields of $50 \mathrm{nT}$ or less, beam-pointing errors can become larger than the beam width, causing loss of track if the error moves the beam off of the $\boldsymbol{B}_{\perp}$-plane. The EDI controller must maintain this accuracy throughout four operational ranges of the FGM data, and this requires constant updates of the four calibration matrices, and four sets of offsets for each axis. As an overall constraint on these numbers, the magnitude of the field is determined by time-of-flight information whenever there are beam hits. As a starting point, the spin-axis offset is adjusted to be consistent with this magnitude. Furthermore, the plane perpendicular to $\boldsymbol{B}$ is determined by the continual series of gun vectors that are successful. But as the beam-width is about one degree, and the tracking algorithm is able to keep the gun pointing only to within about 0.5 degrees of perpendicular to the varying $\boldsymbol{B}$ field, this information must be compiled statistically and used to correct the supplied calibration matrices for accuracy in the EDI coordinate system. This process is iterated by ground processing, and then uplinked to the controller, to improve the success rate of beam hits.

\subsection{Operations}

The complex nature of the EDI operations and data processing has meant a long learning curve before the many control parameters, beam-recognition algorithms, and magnetometer calibrations had been adjusted sufficiently well that the instrument began to operate successfully under a wide range of ambient conditions. More than 15 patches to the onboard software have been uploaded so far. Still, when the magnetic field gets really low, and/or the background electron fluxes get high, tracking becomes difficult. Low $B$ magnitudes require high beam currents to overcome the beam divergence along large gyro orbits, and to get sufficient signal-to-background ratio. But large beam currents, in conjunction with the beam-modulation and -coding lead to interference with the electric wave measurements by the WHISPER instrument (Décréau et al., 1997). Moreover, the smaller $B$ gets, the higher the requirement for very precise on-board magnetometer calibrations. As mentioned in the previous section, improvements in these calibrations are on- 


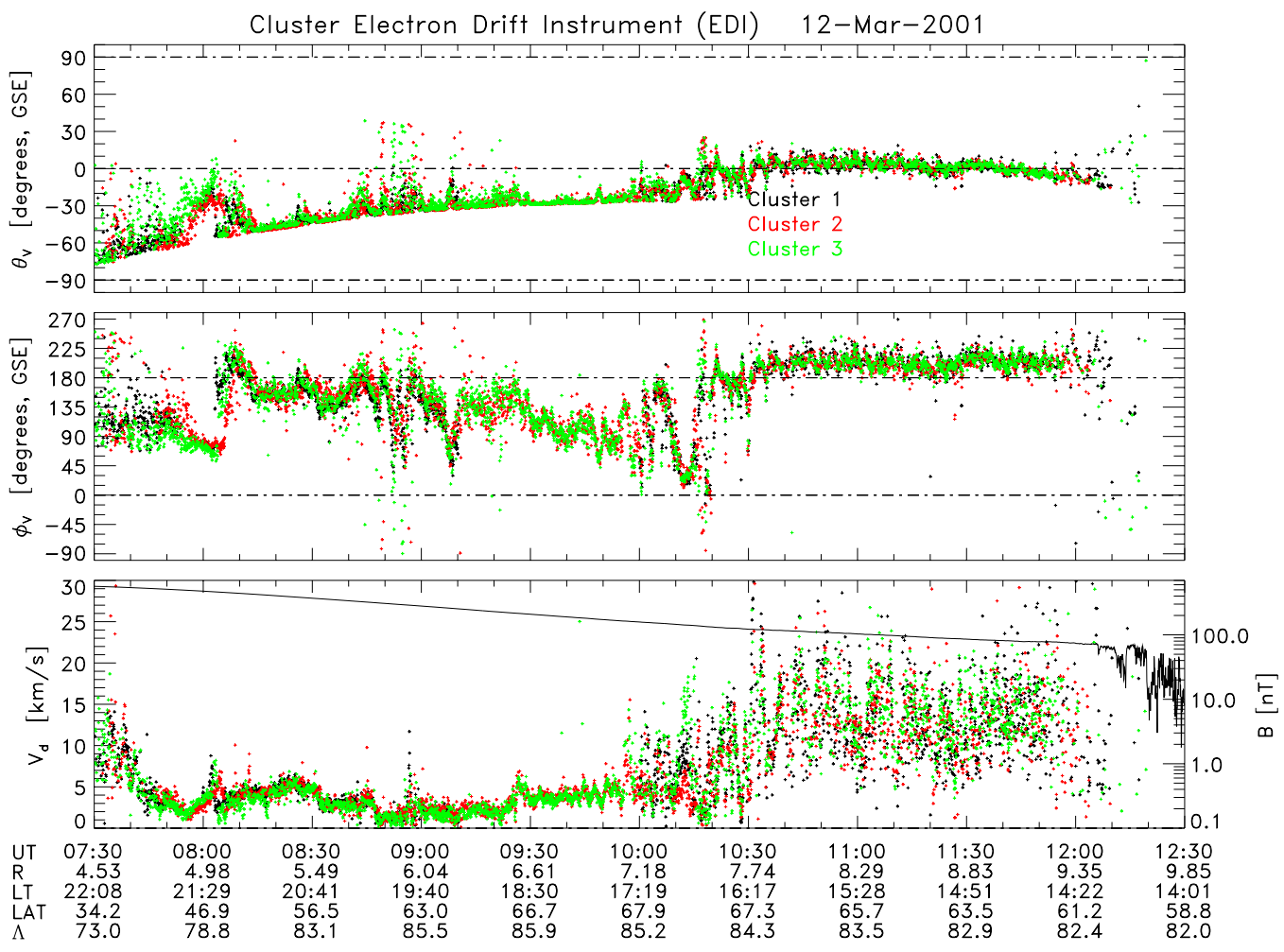

Fig. 7. The same data as in Fig. 6, except that the drift directions are now in GSE and the spacecraft motion has been corrected for.

going. Last but not least, rapid time-variations in magnetic and/or electric fields, as well as large fluxes of background fluxes can also cause loss of track.

\section{Analysis}

\subsection{Data}

EDI sends back the gun firing directions, detector count rates, measured times-of-flight, correlator settings, and some signal-quality information once every telemetry record, i.e. every $128 \mathrm{~ms}$ or $16 \mathrm{~ms}$ in nominal (NM) and burst (BM) telemetry, respectively. Additional auxiliary information (beam currents, optics states, control-loop parameter settings) is transmitted once every telemetry format (5.2 s). As EDI operates asynchronously, time-tags are added to every data record.

Figure 3 shows a 15 -second period on 12 March 2001 that illustrates the character and quality of the raw EDI data when the magnetic field is fairly high, $100 \mathrm{nT}$ in this case, and the flux of background electrons is very low. The spacecraft was transmitting in nominal (NM) mode, which means that an EDI data record is available once every $128 \mathrm{~ms}$. In this example, which shows the data from Gun 2 and Detector 1 on $\mathrm{SC} 3$, beam tracking was successful a large fraction of the time, as evidenced by the high detector counts in the top panel, but more significantly by the high (squared) signalto-noise ratio (SNR) in the second panel, which is computed from the contrast between matched and unmatched correlator channels. Levels of this quantity in excess of the threshold indicated by the lower dashed line mark the times when the beam has been acquired (angle-track). If the signal is kept in correlator number 7 (third panel), this indicates that timetrack has been achieved as well. The occasional low signaland SNR-levels indicate that the target has not been acquired, and only the ambient background electrons are detected. The $\Theta_{2}$ and $\Phi_{2}$ panels illustrate the rapid changes in gun firing directions that are being executed to track the moving target.

Subsequent data processing is determined by the qualitystatus indicator $q_{1}$ (fourth panel) that is transmitted in telemetry: $q=0$ indicates that no beam-signal was acquired within the last $128 \mathrm{~ms}$ ( $16 \mathrm{~ms}$ in BM telemetry); $q=1$ indicates angle-track, $q=2$ indicates angle as well as time-track, $q=3$ in addition requires that the beam returns with an even higher SNR (upper dashed line in the second panel). The $q=0$ data are useful because they provide the count rates from ambient electrons, as discussed in Quinn et al. (2001, this issue). The $q=1$ data (angle-track only) have been ignored for the present analysis. In line with this selection, the times-of-flight in the bottom panel are shown only for those measurements that achieved time-track.

\subsection{Analysis methods}

From the information reported in telemetry, the beam directions and gun positions in spacecraft-sun (SCS) coordinates are computed, based on the Sun Reference Pulse (SRP). Our 


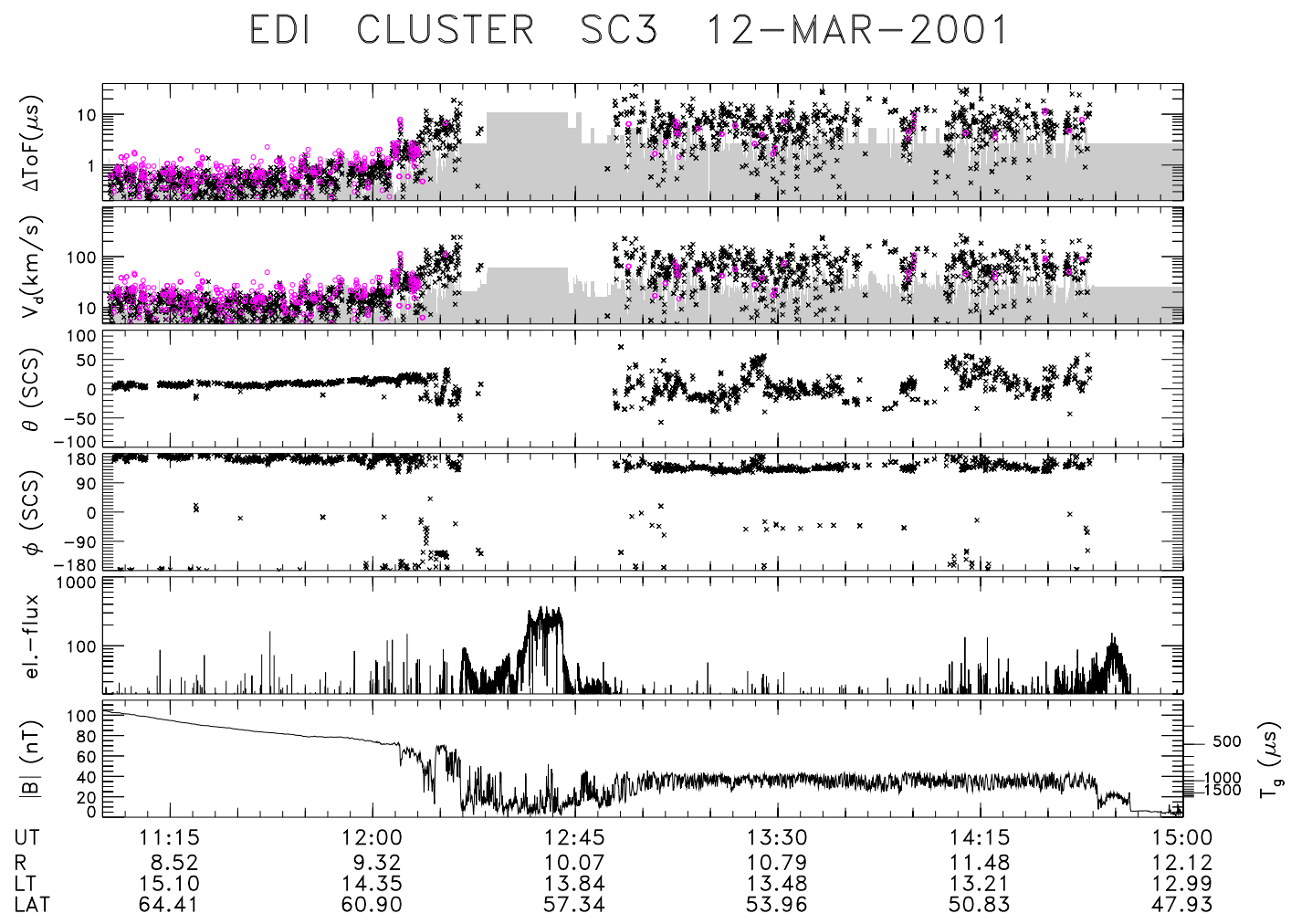

Fig. 8. Drift velocities on 12 March determined from the time-of-flight technique. The top panel shows the difference in the times-of-flight of the two beams, from which the magnitude of the drift velocity (second panel) is directly determined. There are two types of symbols in the top two panels (magenta-coloured circles and black crosses), which refer to the two different techniques to derive the $\Delta T o F s$, as explained in the text. Points in the grey area, which is based on $T_{\text {chip }} / 8$ as the error of the individual measurements, and on the number of points within each 3-spin interval, are not significant. The next two panels show the drift direction. The fifth panel shows the counts (per 2 ms) received from ambient electron fluxes at $1 \mathrm{keV}$ and $90^{\circ}$ pitch-angle, measured by EDI at the times when the beam was not detected. The bottom panel shows the spin-averaged magnetic field strength from FGM for reference. The scale on the right of that panel shows the electron gyro time computed from $B$.

standard analysis is then to select all beams within a certain time-interval, typically one spacecraft spin $(4 \mathrm{~s})$ and to perform an automated determination of the drift step. For higher time resolution analysis, shorter intervals can be chosen (see Quinn et al., 2001, this issue).

\subsubsection{Triangulation analysis}

We have developed an analysis procedure that determines the drift step by searching for the target-point that minimizes an appropriate "cost-function". For each grid-point in the $\boldsymbol{B}_{\perp}$ plane, the cost-function is constructed by adding up the (squared) angle-deviations of all beams in a chosen time interval from the direction to that grid-point. The present software allows selection of $1,1 / 2$ or $1 / 4$ spin period as the analysis interval. Each beam contribution to the cost-function is normalized by the (squared) error in the firing directions, which is a function of beam pointing direction and varies between $1^{\circ}$ and $4^{\circ}$. The grid-point with the smallest value of the cost-function is taken as the target. If a beam has been identified as a multi-runner of order $N$ by the time-offlight analysis (see Sect. 4.2.2), it is associated with a gridpoint at $N$ times the radial distance. When identification of the order from the time-of-flight analysis is ambiguous, there is an alternate method where beams whose firing direction is closer to the direction towards the grid-point at $N$ times the radial distance are counted as runners of order $N$. To speed up the search, the procedure uses a coarse grid to identify a restricted range in which the final search is performed with a much finer grid. The present software approximates the electron trajectories by circles whose radius is based on the magnetic field strength. An example of the drift step determination using this method is shown in Fig. 4. The figure shows gun locations and firing directions for a $4 \mathrm{~s}$ (i.e. one spin) interval during which beams happened to be aimed at the single- and double-runner targets. Note that the construction of the drift step from the firing directions of the beams is for a virtual detector location at the center of the spacecraft. Thus the spacecraft is drawn at twice its actual dimensions, as explained in Quinn et al. (2001, this issue). The red vector from the solid black circle to the center of the spacecraft is the drift step, determined, in the way described above, as the best fit to all the beams in the chosen interval. The figure emphasizes the importance of the correct identification of the multi-runners, in this case double-runners only. The example 


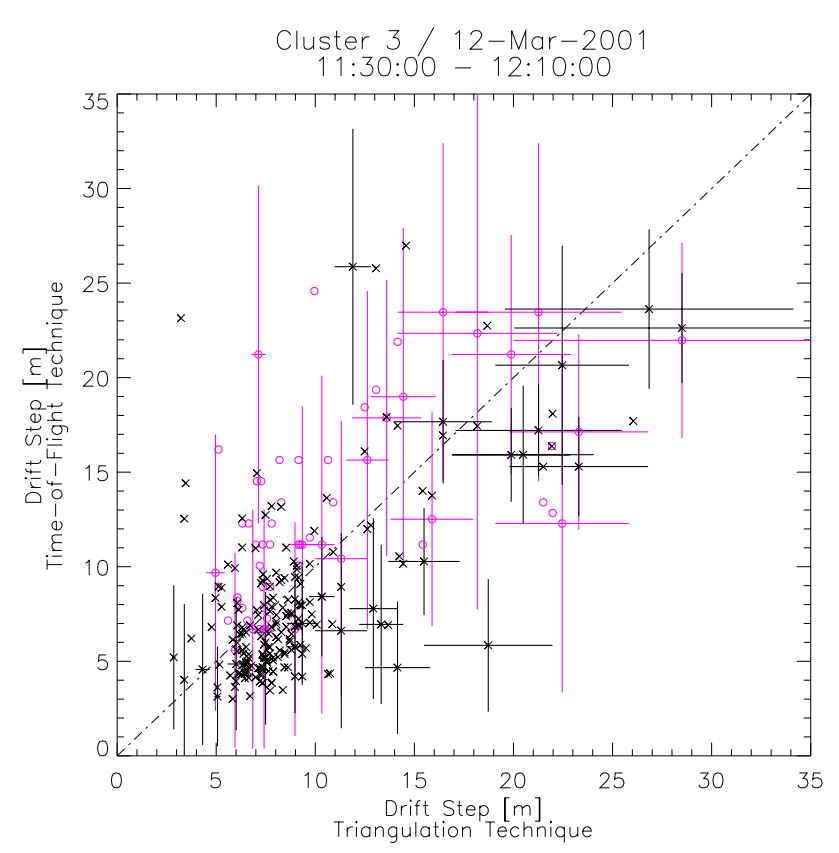

Fig. 9. Scatter plot of the drift speeds derived by the time-offlight analysis (vertical axis) against those from the triangulation analysis (horizontal axis), for the interval 11:30 through 12:10 on 12 March 2001, on SC 3. The two types of symbols refer to the different time-of-flight analysis methods, as in Fig. 8. Error bars are only shown for a fraction of the points so as not to clutter the figure.

is for a less than perfect focus to illustrate the power of the statistical approach of this analysis technique. An example of a much tighter focus is given in Quinn et al. (2001, this issue), Fig. 2. The analysis fails if the drift step and/or the magnetic field significantly vary within the chosen time interval. We can identify such cases by the variance in the magnetic field, by the quality of the fit (as measured by its reduced $\chi^{2}$ ), and by the angle or magnitude errors in the computed drift step. If those quantities exceed certain limits, no output is generated. For the present paper, we have excluded data where the errors in drift step magnitude were larger than $30 \%$, or the reduced $\chi^{2}$ was larger than 20 .

\subsubsection{Time-of-flight analysis}

The time-of-flight analysis serves three purposes. First and foremost, it is used to determine the drift velocity when the drift step becomes too large for the triangulation analysis. Second, it helps to identify multi-runners and thus can support the triangulation analysis, and third, it is equivalent to a measurement of $B$. Deduction of the drift step (and the drift velocity) from analysis of the difference in the times-of-flight of the two beams (Eq. 3) is, in principle, straightforward.

If the drift step is large enough such that the firing directions become nearly parallel, then one can easily group all the beams in the analysis interval (e.g. the spin period) into two oppositely directed sets. The set with the larger timesof-flight then must contain the beams directed towards the target, the other set those directed away from the target. This assignment settles the drift direction, and the drift magnitude is then computed from the magnitude of the difference in the times-of-flight.

This simple scheme requires that conditions are stable over the analysis interval. If this is not the case, one should only use nearly simultaneous towards- and away-beam pairs for the analysis. But as we do not always have simultaneous hits from the two guns, we often have to resort to a method where we take the instantaneous difference between each measured time-of-flight and the gyro-time, $T_{g}$, computed from the high-resolution magnetic field data from FGM. According to Eq. 2, the times-of-flight of the towards- and away-beams are symmetric around $T_{g}$, so that, in principle, either would be sufficient to compute the magnitude of the drift. But because the times-of-flight differ from $T_{g}$ by a percent at most, this scheme would work only if $T_{g}$ were known precisely. In practice, the $T_{g}$ computed from the actual magnetic field measurements, $T_{g, \text { est }}$, will not be properly centered, and we therefore cannot apply this scheme directly. Instead, we average the differences between any measured time-of-flight and the corresponding $T_{g, e s t}$ separately for the two sets of beams. This way any fixed magnitude offset in $T_{g \text {,est }}$ will cancel out. The set with the larger average is identified with the towardsbeams, the other with the away-beams, as above. The difference between the two averages is then the quantity to use for $\Delta T$ in Eq. 3. The identification of multirunners from the times-of-flight is illustrated in Fig. 5, which shows an example of the measured times-of-flight for Detector 1 for a onehour interval on 12 March. The red x's are the hits identified as single-runners. The other traces are from multi-runners, as identified in the legend. As described earlier, the PN-code is much shorter than the gyro time. In this particular case $T_{g}$ varies between 350 and $480 \mu$ s, while the code length remains fixed at $114.4 \mu \mathrm{s}$. The electrons having gyrated twice have therefore an apparent increment in time-of-flight of $T_{g}$ modulo $114.4 \mu$ s relative to the single-runners. The same increment applies to each higher multiple. Increments that are larger than half the code-length lead to apparent multirunner times-of-flight that are actually smaller, as seen in Fig. 5. Applying this simple rule one can then identify the multirunner order $N$. Note that the slope of the multi-runner traces is $N$ times that of the single runners. Naturally, this method fails when $T_{g}$ is itself a multiple of the code-length. In the example at hand this condition occurs where the multi-runner traces intersect, near the beginning and end of the interval shown. As mentioned earlier, a by product of the EDI timeof-flight measurements is that they provide a precise determination of the magnetic field magnitude. Data such as shown in Fig. 5 have been provided routinely to the FGM team to validate the spin-axis offsets in the FGM calibrations.

\section{Results}

In the following we present spin-resolution EDI data for three outbound passes when the apogee was located near local noon. During these orbits, EDI was not operated for approxi- 


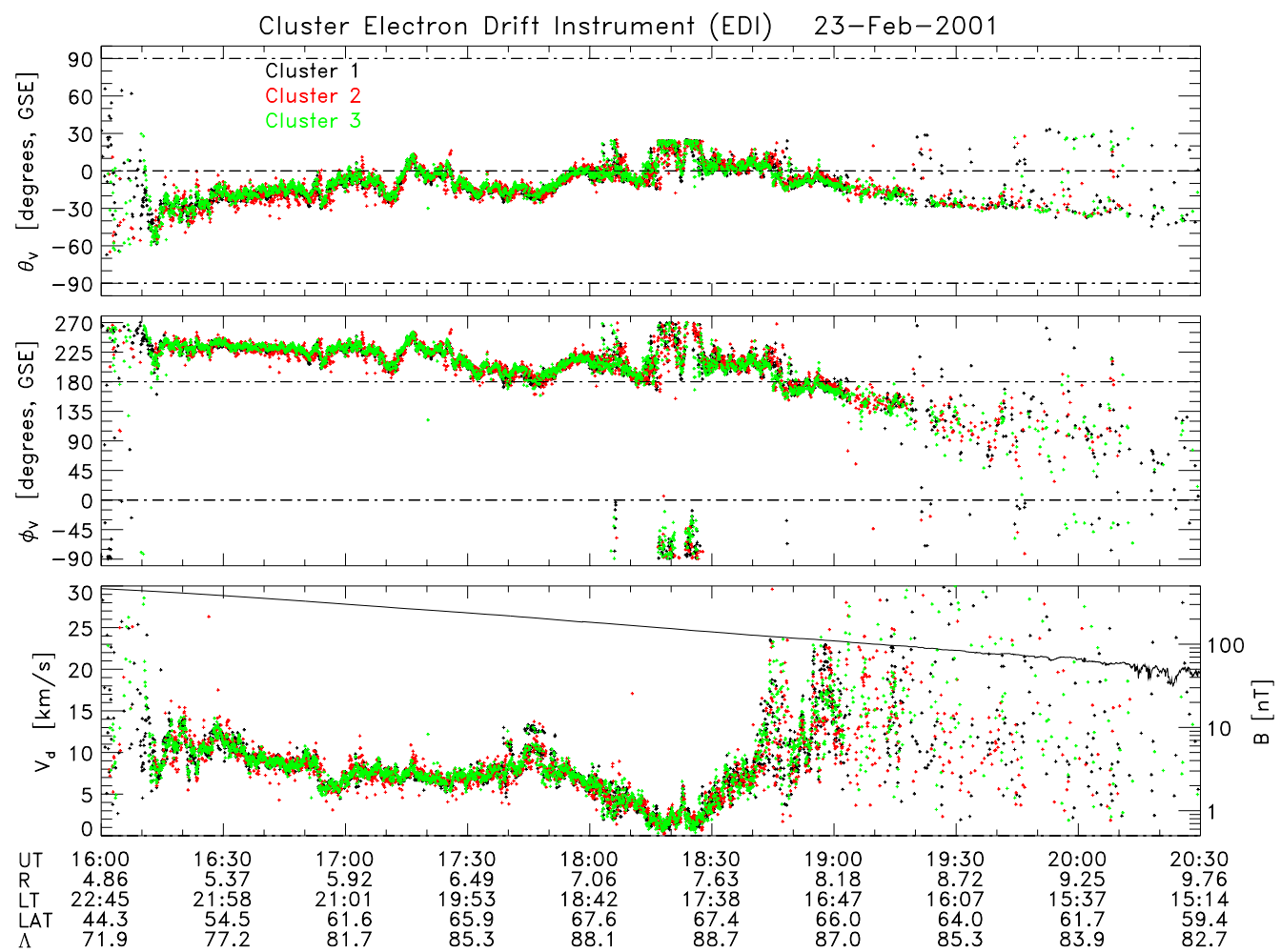

Fig. 10. Outbound pass on 23 February 2001, in the same format as Fig. 7, i.e. with the spacecraft velocity corrected for.
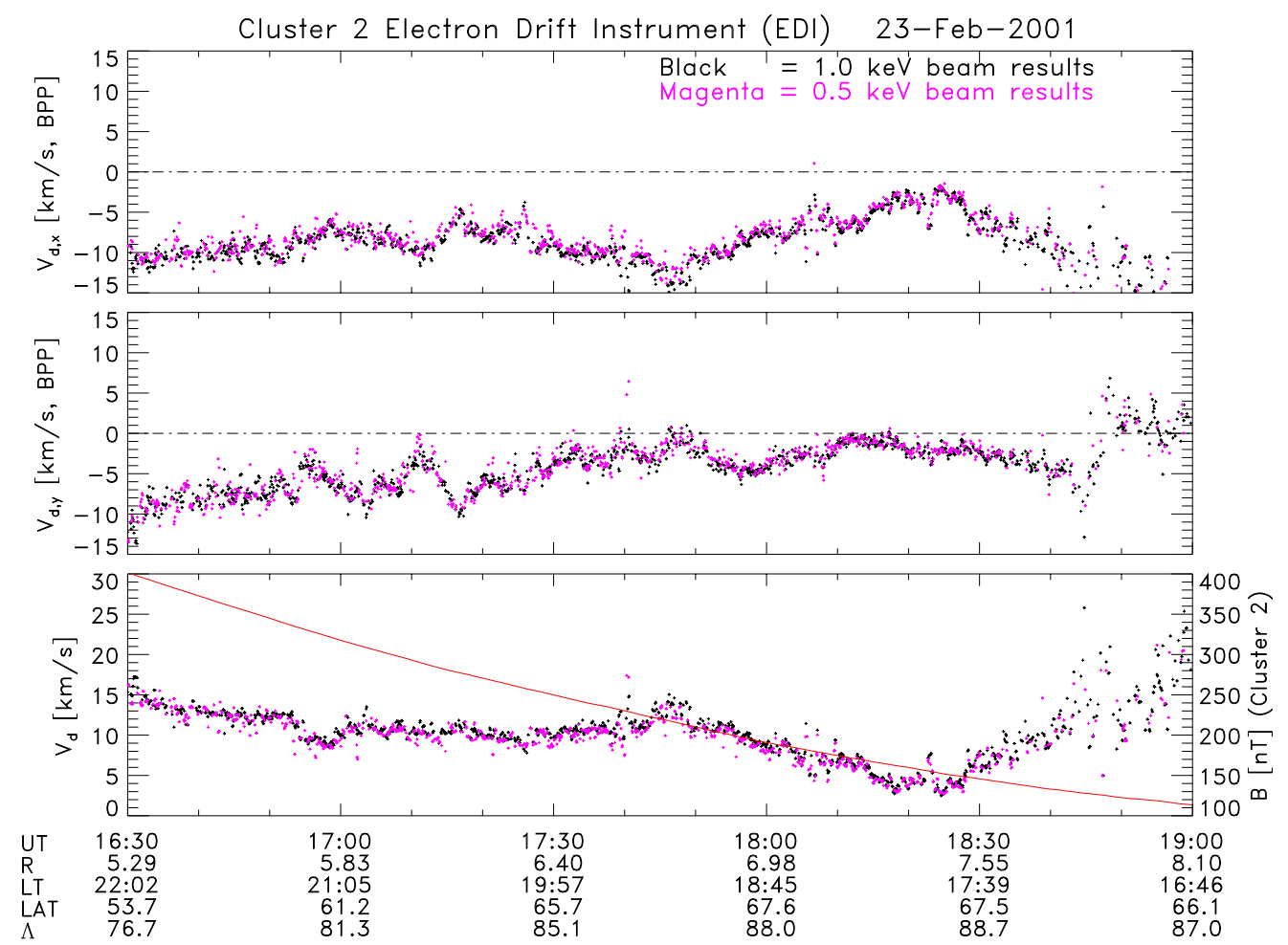

Fig. 11. Comparison of the drift velocities measured at 0.5 and $1.0 \mathrm{keV}$ for the pass on 23 February, 16:30-19:00 UT. The plot shows the two components of the drift in the $\boldsymbol{B}_{\perp}$-plane, plus the magnitude. The magnitude of $B$ is shown superimposed in the bottom panel. 


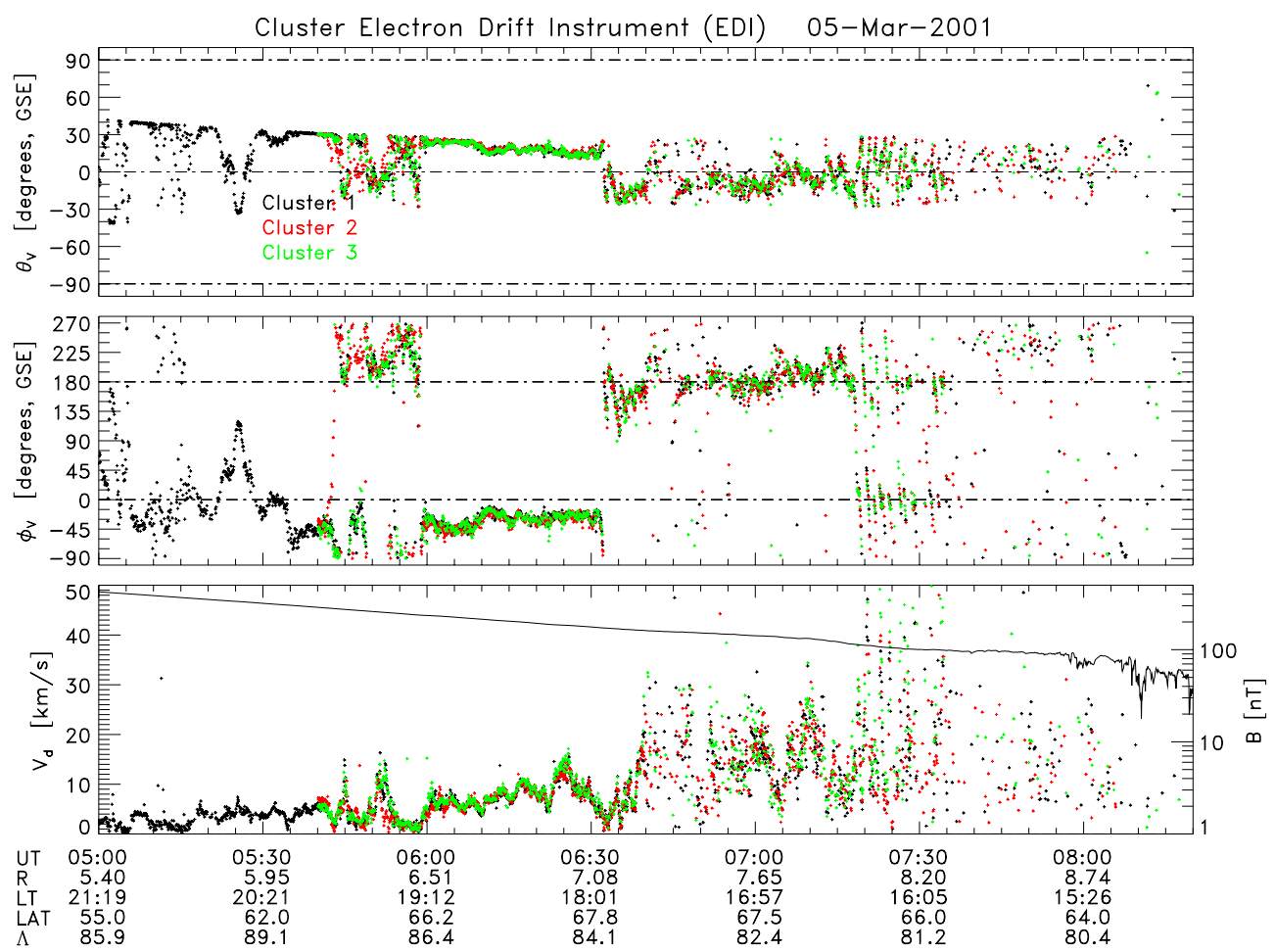

Fig. 12. Outbound pass on 5 March 2001 in the same format as Fig. 7. Note that until 05:40 only SC 1 data are available.

mately a 2-hour period centered on perigee. In addition, EDI was not operated on SC 4 during these orbits because of some intermittent overcurrent condition.

The EDI data are presented either as drift velocities or as electric fields, and in one of three coordinate systems. One is the spacecraft-sun (SCS) system, the second is the geocentric solar ecliptic (GSE) system, and the third is the $\boldsymbol{B}_{\perp}$-plane system. The SCS system has its $Z$-axis along the spacecraft spin axis (directed nearly along the $-Z_{\mathrm{GSE}}$ axis), and its $X$ axis directed sunward and is thus the system in which most Cluster instruments acquire their measurements. The GSE system has the advantage that it is an inertial system that allows to judge the drift direction in absolute terms. To be consistent with this inertial nature, we correct for the spacecraft velocity when showing data in GSE. The $\boldsymbol{B}_{\perp}$-plane (BPP) system, on the other hand, is a natural system for EDI because it emphasizes the fact that the measurements are twodimensional in nature. It has the disadvantage that its axes change direction as $\boldsymbol{B}$ changes. The BPP-system has its $X$ axis directed towards the sun (more precisely $X$ is in the plane containing $\boldsymbol{B}$ and the sun) and its $Z$-axis such that it has a positive $Z_{\mathrm{GSE}}$ component.

\subsection{Outbound pass on 12 March 2001}

Figure 6 shows the drift velocities, measured on spacecrafts 1, 2 and 3, for the outbound pass on 12 March 2001 from 07:30 to 12:30 UT, obtained with the triangulation method (Sect. 4.2.1). The data start at $4.5 R_{E}$ at $34^{\circ}$ GSE- latitude, $73^{\circ}$ invariant latitude, and 22 hours local time, i.e. on magnetic field lines connected to the high-latitude edge of the nightside auroral oval. The orbit then crosses the northern polar cap and exits the magnetosphere at about $59^{\circ}$ latitude at 12:10 UT, as determined from the sudden drop in $B$ shown in the third panel. The drift velocities are presented in the SCS system and not corrected for spacecraft velocity, to emphasize what is observed in the spacecraft system. The figure shows that there is very good overall agreement between the measurements on the three spacecraft, particularly regarding the drift directions. A proper interpretation of the drift velocities requires the data to be put into an inertial frame. Figure 7 therefore shows the same data, but now in GSE coordinates and corrected for the spacecraft motion. This correction means adding to the measured drift velocity the perpendicular component of the spacecraft velocity, because the motion of the spacecraft through the plasma implies a drift in the opposite sense. While the transition from SCS to GSE is simply a rotation, effectively flipping the signs of both angles, the correction for the spacecraft velocity has a dramatic effect, both in direction and magnitude. This is because in large parts of the pass the spacecraft velocity is of similar magnitude as the drift velocity, and furthermore both velocities are directed nearly opposite to each other some of the time. This means that the magnitude of the drift velocity can become very small after the correction, and its direction not only can become quite different, but also less well defined. This explains why the directions in Fig. 7 are much more variable much of the time than in Fig. 6. Note that the 


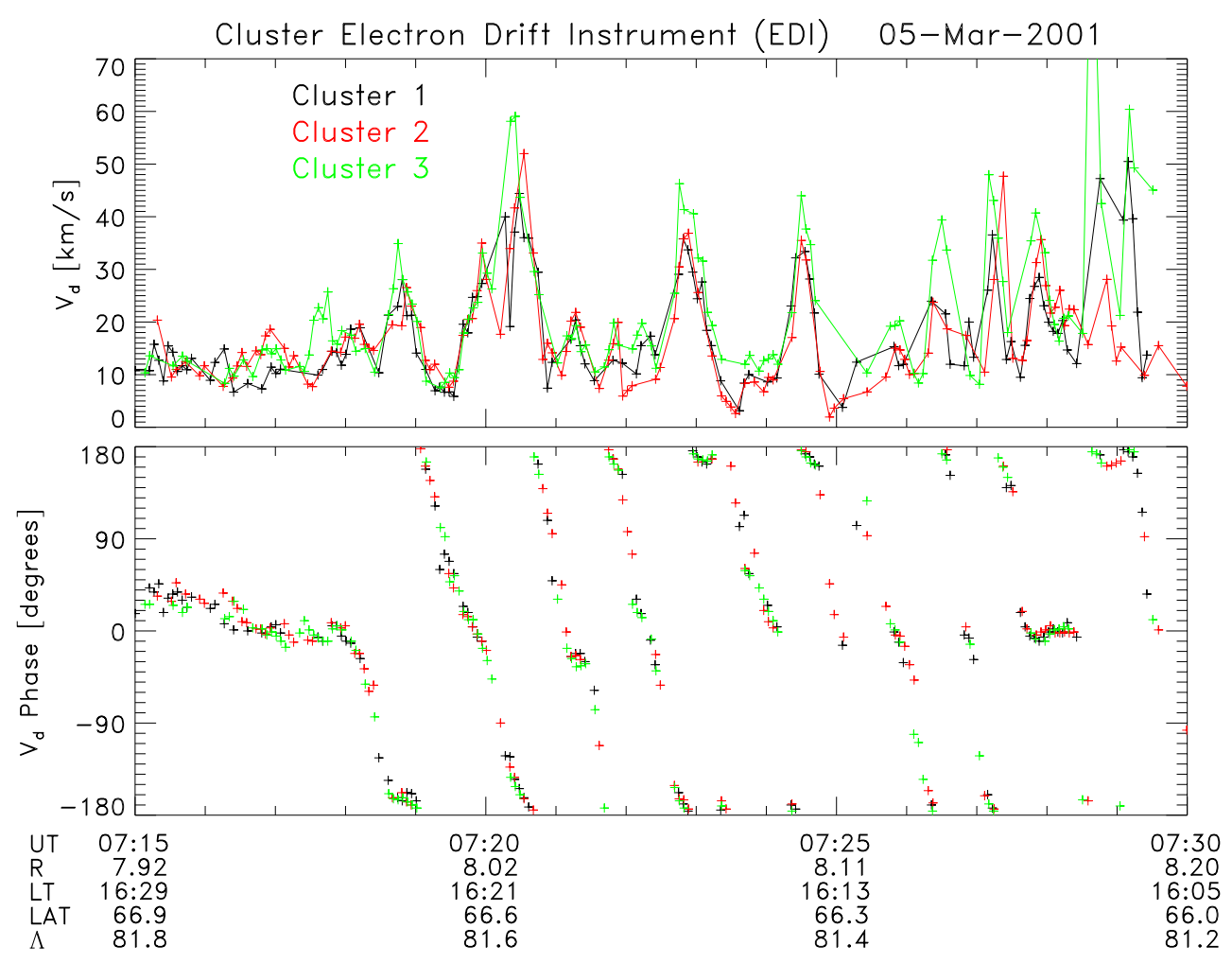

Fig. 13. Drift velocity magnitude and phase in the gyro plane, for $07: 15$ to $07: 30$ on 5 March, demonstrating full $360^{\circ}$ rotations of the drift velocity vector.

triangular feature in the flow azimuth near 08:00 in Fig. 6 has now become a similarly looking feature in the drift magnitude. As the drift velocity is, by definition, constrained to the $\boldsymbol{B}_{\perp}$-plane, its possible directions are restricted by the magnetic field orientation. This explains the sometimes clipped appearance of the angle-traces in this and the following figures.

Figure 7 shows that after exiting the auroral flux tubes with their fairly high but variable convection velocities, the drift velocity stays low $\left(<5 \mathrm{~km} \mathrm{~s}^{-1}\right)$ until 10:00 UT, with directions ranging from anti-sunward to almost sunward. The dips in drift speed near 09:00 correspond to electric fields as low as $0.1 \mathrm{mV} \mathrm{m}^{-1}$, which highlights the sensitivity of the EDI measurements. Near 10:00, the convection speed suddenly becomes larger $\left(10-20 \mathrm{~km} \mathrm{~s}^{-1}\right)$ and highly variable, with equally variable directions, but the direction soon (at 10:30) settles on a stable, essentially anti-sunward direction $\left(\Phi_{v}\right.$ near $180^{\circ}, \Theta_{v}$ near $0^{\circ}$ ). There are only a few measurements after 12:00 UT, i.e. when approaching the magnetopause, and their validity is questionable because of increasing time variations within the analysis interval.

Figure 8 shows the measurements for the same day from 11:00 UT up to the bow shock, which is crossed at 14:48 UT. In this case the drifts were determined from the $\Delta T o F s$, the difference in the measured times-of-flight of the two beams, shown in the top panel. There are two types of points (in this and the second panel). The open magenta-coloured circles were directly computed from the times-of-flight of the two beams for those cases when they hit their detectors nearly simultaneously, within $\pm 15 \mathrm{~ms}$ in this case. Those $\triangle T o F s$ were then averaged over a three-spin interval that slides along one spin at a time. There are only few such points after 13:00 UT, because the number of hits was getting much smaller there and so did the likelihood of having near-simultaneous ones. The black crosses are also 3-spin averages, but were obtained by the other method described in Section 4.2.2, where the gyro-times estimated from the FGM data serve as intermediate reference. The agreement between the two sets of points is quite good.

The second panel shows the drift speeds computed from the $\Delta T o F s$ according to Eq. 3, the third and fourth panels the drift directions. The drift directions are derived from the beam pointing directions and the time-of-flight analysis that involves the magnetic field as reference, thus the crosses. Only those directions are shown for which the drift magnitude is significant, i.e. outside the grey area. In spite of this restriction, there are a few points left whose direction is opposite to those of the others. These represent cases where the inferred $\triangle T o F s$ apparently had the wrong sign.

Until about 12:00, the time-of-flight differences are of order $1 \mu \mathrm{s}$, compared to a code-chip length of $7.6 \mu \mathrm{s}$, and therefore are just barely detectable. This explains the fairly large scatter in the derived drift speeds, whose magnitude is about $20 \mathrm{~km} \mathrm{~s}^{-1}$ on average. Note that at this time the HIA sensor of the CIS instrument measures a bulk velocity component perpendicular to $\boldsymbol{B}$ of $20 \mathrm{~km} \mathrm{~s}^{-1}$, in good agreement with the 
EDI measurements (B. Klecker, private communication).

The drift direction is well defined and stable, almost precisely anti-sunward. At 12:05 the drift speed picks up and the direction becomes variable, until tracking stops at 12:20 because the magnetic field magnitude drops to $5 \mathrm{nT}$, indicating the crossing of the magnetopause. Such field strengths are prohibitively low for the EDI technique because of the $B^{3}$ dependence of the flux that returns to the detector (see Sect. 3.1).

In the magnetosheath proper, i.e. after 13:00 UT, the $\Delta T o F s$ rise to $10 \mu \mathrm{s}$ on average, if one discounts the points in the grey area, corresponding to about $100 \mathrm{~km} \mathrm{~s}^{-1}$, and the convection is essentially anti-sunward, in good agreement with the perpendicular component of the bulk velocity measured by HIA. But as the chip-length has risen to $30 \mu \mathrm{s}$, the accuracy is not much better than before 12:00. Furthermore, the magnetic field is now highly variable, and this introduces extra scatter.

Comparing the measured $\triangle T o F s$ with the gyro times shown by the scale to the right of the bottom panel, it is apparent that the times-of-flight deviate by only $1 \%$ or less from the gyro times, which highlights the measurement problem. We are still working on optimizing the time-offlight measurement accuracy, by reducing the chip-length, while at the same time maintaining adequate tracking capability and avoiding the ambiguities inherent in the use of short code-lengths, discussed in Sect. 3.2.

Figure 8 also illustrates another aspect that affects EDI operation. The next to last panel shows the counts from ambient electron fluxes at $1 \mathrm{keV}$ energy and $90^{\circ}$ pitch-angle, measured by EDI at the times when the beams are not detected (identified by the quality status $q=0$ ). High fluxes are observed just outside the magnetopause and near the bow shock, in agreement with measurements by the PEACE instrument (A. Fazakerley, private communication). To detect the beams in the presence of such high ambient fluxes would require very high beam currents. In spite of these limitations, Fig. 8 demonstrates that EDI is able to continuously track across the magnetosheath in magnetic fields that are as low as $30 \mathrm{nT}$ and as variable as is typical for the magnetosheath. EDI stops tracking at 14:40 UT, i.e. shortly before the bow shock crossing, presumably because beam currents were limited to about $100 \mathrm{nA}$ at the time, and the fluxes of background electrons became high. Between 11:30 and 12:10 on this pass, the triangulation and time-of-flight analysis methods have both provided results. In Fig. 9 we have plotted the drift steps obtained by the two methods against each other. Most of the points come from times before 12:00 where the drift steps are less than $10 \mathrm{~m}$, and thus difficult for the time-of-flight technique to resolve. Nevertheless, the figure shows that within the admittedly often large errors, there is reasonable agreement between the two techniques.

\subsection{Outbound pass on 23 February 2001}

Figure 10 shows an overview of the outbound pass on 23 February 2001 from 16:00 to 20:30 UT. At the beginning the

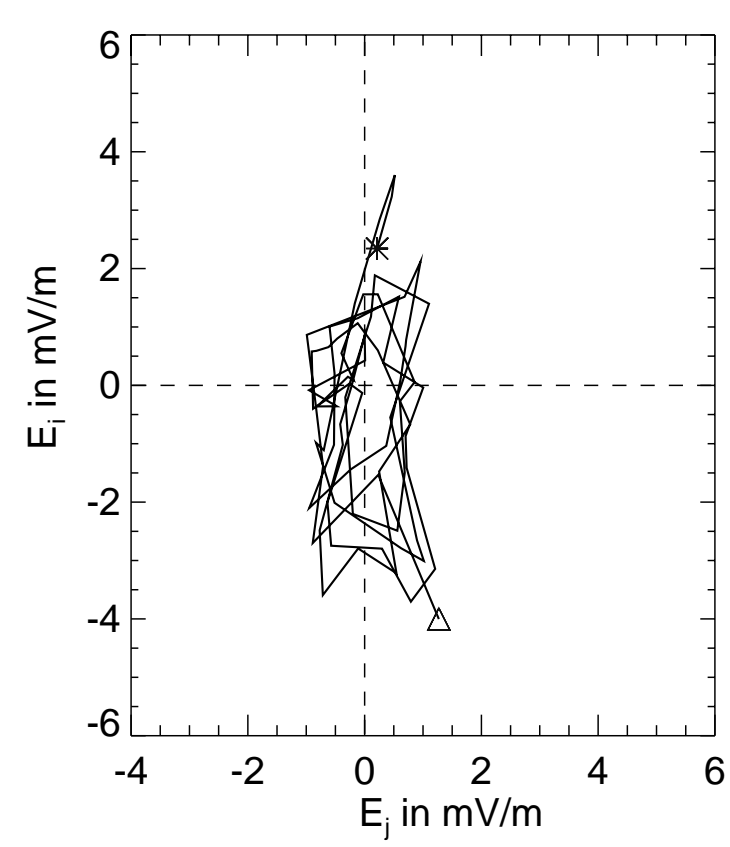

Fig. 14. Hodogram of the electric field measured on SC 3 for the event from Fig. 13, illustrating the elliptical polarization of the oscillations.

spacecraft are at $4.9 R_{E}, 44^{\circ}$ GSE-latitude and $72^{\circ}$ invariant latitude on auroral field lines, proceed across the northern polar cap and exit the magnetosphere near 20:20 UT, at 9.7 $R_{E}$ and $60^{\circ}$ latitude in the afternoon sector. The orbit is similar to that for the 12 March pass, and so are many of the observed features, notably the variable drifts on auroral field lines, and the predominance of anti-sunward convection at typically 5$10 \mathrm{~km} \mathrm{~s}^{-1}$. Near 18:20 the magnitude of the drift velocity (after correction for the spacecraft velocity) becomes very low, less than $1 \mathrm{~km} \mathrm{~s}^{-1}$, corresponding to electric fields of only $0.1 \mathrm{mV} \mathrm{m}^{-1}$. The drift speed then picks up on average, but is highly variable. On approach to the magnetopause , i.e. after 19:20, there are occasional flips in drift direction by $180^{\circ}$, from anti-sunward to sunward, which are not real and are probably due to variations occurring on spin-period timescales. During part of this orbit, EDI on SC 2 was operated such that the electron energy was switched between 0.5 and $1.0 \mathrm{keV}$ every second. Figure 11 shows that the drift velocities measured at the two energies agree to within less than $0.5 \mathrm{~km} \mathrm{~s}^{-1}$ most of the time, implying that the $\nabla B$ drift (see Eq. 7) was essentially zero, and the drift velocities measured at the two energies thus both represent the true $\boldsymbol{E} \times \boldsymbol{B}$ drift. More precisely, if one considers $0.5 \mathrm{~km} \mathrm{~s}^{-1}$ to be the upper limit for the difference in drift velocities in this example, then according to Eq. 6, with $B=200 \mathrm{nT}$, one gets $10000 \mathrm{~km}$ as the lower limit for the gradient scale length in the magnetic field at this time. If the gradient scale length had been larger, the drift velocities at the two energies would have become significantly different. The capability to separate the $\boldsymbol{E} \times \boldsymbol{B}$ and $\nabla B$ drifts is a unique feature of EDI and can be used to determine local magnetic field gradients if the induced drift 

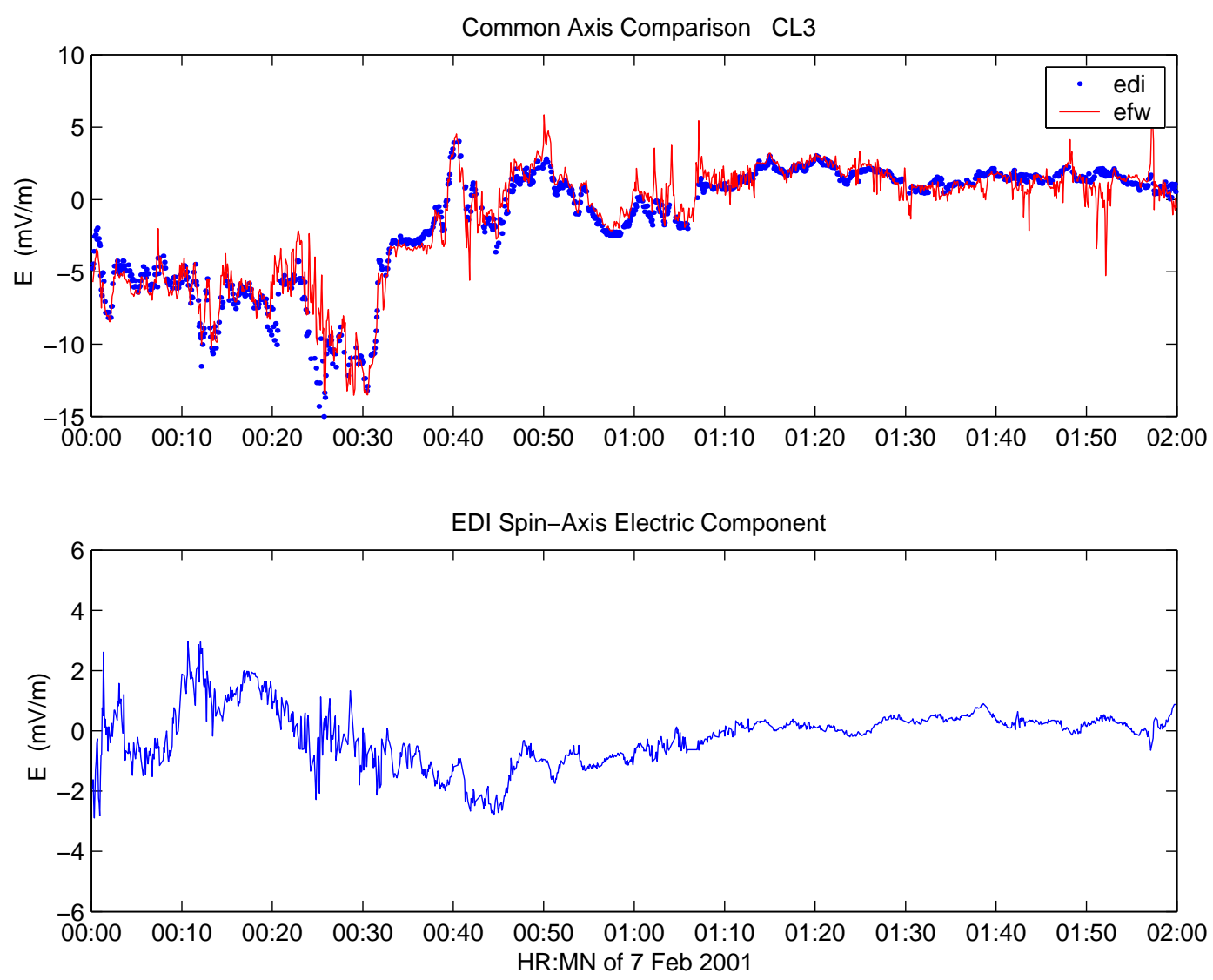

Fig. 15. Comparison of EDI and EFW electric fields. The top panel shows the component of $\boldsymbol{E}$ along the common axis defined in the text. The bottom panel shows the spin axis component measured by EDI.

is strong enough to compete with the electric field drift. So far we have not yet obtained measurements in regions where one expects the $\nabla B$ drift to contribute measurably to the total drift.

\subsection{Outbound pass on 5 March 2001}

An interesting event has been observed during the outbound pass on 5 March 2001. Figure 12 shows the drift velocities starting at 05:00 UT, when the spacecraft are near 5.4 $R_{E}$ at $55^{\circ}$ latitude $\left(86^{\circ}\right.$ invariant latitude) and then proceed over the polar cap to the magnetopause, which is crossed after 08:00 UT. Until 06:30, drift speeds slowly increase from less than 1 to $10 \mathrm{~km} \mathrm{~s}^{-1}$, with highly variable directions, but overall good agreement between the spacecraft. The low speeds just before 06:00 and after 06:30 correspond to electric fields of $0.1 \mathrm{mV} \mathrm{m}^{-1}$. There is a period (until about $07: 15$ ) when the drift velocities become highly variable in magnitude, but are predominantly anti-sunward. Starting at 07:18, largeamplitude oscillations are observed in direction and magnitude, with periods near 1 to $1.5 \mathrm{~min}$. Focusing on the interval 07:15 to 07:30 UT, and presenting the data as magnitude and phase of the drift velocity in the $\boldsymbol{B}_{\perp}$-plane, Fig. 13 shows that the drift vector performs many full $360^{\circ}$ rotations during the event. The agreement between the three spacecraft is remarkable, and there is no discernible time-displacement either, implying a structure that is homogeneous over a scale that exceeds the spacecraft separations, which range from 425 to $840 \mathrm{~km}$. The hodogram of the equivalent electric field shown in Fig. 14 shows a very elliptical, left-handed polarization of the oscillations. The hodogram appears offset from the origin because of some net background drift velocity.

\subsection{Comparison with EFW}

As already stated in Sect. 1, the EDI and EFW instruments complement each other in that both directly or indirectly measure the electric field, but are subject to different kinds of limitations. It is therefore of great importance that the measurements are first compared under conditions when both should return valid electric field measurements. Figure 15 shows such a comparison on SC 3 on 7 February 2001. As EFW measures the field in the spin-plane, while the EDI measurements are in the $\boldsymbol{B}_{\perp}$-plane, the figure (top panel) compares the electric fields along the common axis defined by the intersection of the two planes. The bottom panel shows the spin-axis component of the electric field that is measured by EDI but not by EFW, and that can often be a significant part of the total field. As the figure shows, the measurements along the common axis agree remarkably well 
in this case, for which that axis is almost transverse to the earth-sun line. We are presently studying some occasions, when the common axis is more nearly aligned with the earthsun line, or when there are rapid excursions of the plasma density to very low values, where the agreement is usually not so good. The large electric fields observed in this case, which when mapped into the ionosphere are of the order of $100 \mathrm{mV} \mathrm{m}^{-1}$, correspond to the onset of a substorm on this date, as seen by EFW and EDI on spacecrafts 1,2 and 3. This will be the subject of a later publication.

\section{Summary}

In this paper we have presented three polar passes that demonstrate that EDI is able to make precise drift velocity measurements under a wide range of conditions, which include the low and variable magnetic and electric fields in the magnetosheath. Drift velocities as low as $1 \mathrm{~km} \mathrm{~s}^{-1}$ are observed, corresponding to electric fields of $0.1 \mathrm{mV} \mathrm{m}^{-1}$. An outstanding feature in these observations is the quasiperiodic electric field rotationsobserved on 5 March 2001 over the polar cap on the dayside at $81^{\circ}$ invariant latitude. A key advantage of the EDI technique is that the beam probes the ambient electric field at a distance of some kilometers from the spacecraft, and therefore essentially outside the latter's influence. Furthermore, the analysis is essentially geometric in nature and thus the accuracy can be quite high. And last but not least, EDI always measures the entire drift velocity, and thus the total transverse electric field, including any component along the spacecraft spin axis, while the double-probe instrument on Cluster (EFW) measures only in the spin-plane. On the other hand, EDI beam tracking will be disrupted in very low magnetic fields, large fluxes of ambient electrons, and by very rapid changes in magnetic and/or electric fields. Thus EDI and EFW complement each other nicely. Comparisons with EFW are turning out to be very promising, as the remarkable agreement in the example presented in this paper demonstrates. Comparisons with the perpendicular component of the plasma bulk velocity measured by the CIS instrument have also started.

A unique feature of EDI is its capability to separate the $\boldsymbol{E} \times \boldsymbol{B}$ and $\nabla B$ drifts that we have demonstrated with one example in this paper (where the $\nabla B$ drift happened to be essentially zero). This capability could be used to determine magnetic field gradients over the distance of the electron gyro radius, thus complementing the technique to infer the gradients over a much larger scale from the magnetic field measurements on the four spacecraft.

Acknowledgement. The authors would like to thank the many people who have made the EDI experiment possible through 15 years of development, testing, and operations: B. Austin, B. Briggs, J. Chan, M. Chutter, R. Frenzel, W. Göbel, J. Googins, D. Hallmark, W. Isaac, G. King, S. Longworth, K. Lynch, R. Maheu, F. Melzner, J. Needell, U. Pagel, P. Parigger, D. Simpson, W. Stöberl, J. Stöcker, K. Strickler, and C. Young. We owe great thanks to the payload team at Dornier, in particular Roland Nord, and to the entire project team at ESTEC. We acknowledge the excellent operations support by the project scientists at ESTEC, Philippe Escoubet and Michael Fehringer, and by the team at ESOC, in particular Sandro Matussi and Michael Schmidt. EDI could not be operated without the accurate on-board magnetometer data from FGM and STAFF, and we are grateful to Andre Balogh and Nicole Cornilleau-Wehrlin and their teams for the cooperative support they have provided. We thank Forrest Mozer and the EFW team for their efforts on the EDI-EFW comparisons. Joshua Semeter gave advice concerning the analysis technique. We dedicate this paper to the memory of Norbert Sckopke, who contributed so much to the success and spirit of Cluster and EDI, but did not live to see the result. This work was supported by DLR through grants FKZ: 50OC89043 and FKZ: 50OC9705, and by NASA through grants NAS5-30744 and NAG5-9960.

The Editor in Chief thanks D. Fontaine and F. Lefeuvre for their help in evaluating this paper.

\section{References}

Balogh, A., Dunlop, M. W., Cowley, S. W. H., Southwood, D. J., Thomlinson, J. G., Glassmeier, K. H., Musmann, G., Lühr, H., Buchert,S., Acuña, M. H., Fairfield, D. H., Slavin, J. A., Riedler, W., Schwingenschuh, K., and Kivelson, M. G.: The Cluster Magnetic Field Investigation, Space Sci. Rev., 79, 65-191, 1997.

Cornilleau-Wehrlin, N., Chauveau, P., Louis, S., Meyer, A., Nappa, J. M., Perraut, S., Rezeau, L., Robert, P., Roux, A., De Villedary, C., De Conchy, Y., Friel, L., Harvey, C. C., Hubert, D., Lacombe, C., Manning, R., Wouters, F., Lefeuvre, F., Parrot, M., Pincon, J. L., Poirier, B., Kofmann, W., and Louarn, Ph.: The Cluster Spatio-Temporal Analysis of Field Fluctuations (STAFF) Experiment, Space Sci. Rev., 79, 107-136, 1997.

Décréau, P. M. E., Fergeau, P., Krasnosel'skhikh, V., Léveque, M., Martin, Ph., Randriamboarison, O., Sené, F. X., Trotignon, J. G., Canu, P., and Mögensen, P. B.: WHISPER, a resonance sounder and wave analyser: performances and perspectives for the Cluster mission, Space Sci. Rev., 79, 157-193, 1997.

Gustafsson, G., Boström, R., Holback, B., Holmgrn, G., Lundgren, A., Mozer, F. S., Pankow, D., Harvey, P., Berg. P., Ulrich, R., Pedersen, A., Schmidt, R., Butler, A., Franssen, A. W. C., Klinge, D., Fälthammar, C.-G., Lindqvist, P.-A., Christenson, S., Holtet, J., Lybekk, B., Sten, T. A., Tanskanen, P., Lappalainen, K., and Wygant, J.: The electric field and wave experiment for the Cluster mission, Space Sci. Rev., 79, 137-156, 1997.

Melzner, F., Metzner, G., and Antrack, D.: The Geos electron beam experiment, Space Sci. Rev., 4, 45, 1978.

Nakamura, M., Hayakawa, H., and Tsuruda, K.: Electric field measurement in the ionosphere using the time-of-flight technique, J. Geophys. Res., 94, 5283-5291, 1989.

Paschmann, G., Melzner, F., Frenzel, R., Vaith, H., Parigger, P., Pagel, U., Bauer, O. H., Haerendel, G., Baumjohann, W., Sckopke, N., Torbert, R. B., Briggs, B., Chan, J., Lynch, K., Morey, K., Quinn, J. M., Simpson, D., Young, C., McIlwain, C. E., Fillius, W., Kerr, S. S., Maheu, R., and Whipple, E. C.: The Electron Drift Instrument for Cluster, Space Sci. Rev., 79, 233-269, 1997.

Paschmann, G., McIlwain, C. E., Quinn, J. M., Torbert, R. B., and Whipple, E. C.: in: The electron drift technique for measuring electric and magnetic fields, (Eds) Pfaff, R. F., Borovsky, J. E. and Young, D. T., Measurement Techniques in Space Plasmas: Fields, 29-38, Geophysical Monograph 103, AGU, Washington, D. C., 1998. 
Paschmann, G., Sckopke, N., Vaith, H., Quinn, J. M., Bauer, O. H., Baumjohann, W., Fillius, W., Haerendel, G., Kerr, S. S., Kletzing, C. A., Lynch, K., McIlwain, C. E., Torbert, R. B., and Whipple, E. C.: EDI electron time-of-flight measurements on Equator-S, Ann. Geophysicae, 17, 1513-1520, 1999.

Quinn, J. M., Paschmann, G., Sckopke, N., Jordanova, V. K., Vaith, H., Bauer, O. H., Baumjohann, W., Fillius, W., Haerendel, G., Kerr, S. S., Kletzing, C. A., Lynch, K., McIlwain, C. E., Torbert, R. B., and Whipple, E. C.: EDI convection measurements at 5$6 R_{E}$ in the post-midnight region, Ann. Geophysicae, 17, 1503$1512,1999$.

Quinn, J. M., Paschmann, G., Torbert, R. B., Vaith, H., McIlwain, C. E., Haerendel, G., Bauer, O. H., Bauer, T., Baumjohann, W., Fillius, W., Förster, M., Frey, S., Georgescu, E., Kerr, S. S., Kletzing, C. A., Matsui, H., Puhl-Quinn, P., and Whipple, E. C.: Cluster EDI convection measurements across the high-latitude plasmasheet boundary at midnight, Ann. Geophysicae, 19, this issue, 2001.

Tsuruda, K., Hayakawa, H., and Nakamura, M.: in: Science Objectives of the Geotail Mission, (Ed) Nishida, A., 234, ISAS, Tokyo, 1985.

Tsuruda, K., Hayakawa, H., and Nakamura, M.: Electric field measurements in the magnetosphere by the electron beam boomerang technique, in: Measurement Techniques in Space Plasmas: Fields, (Eds) Pfaff, R. F., Borovsky, J. E., and Young, D. T., 39-45, Geophysical Monograph 103, AGU, Washington, D. C. , 1998.

Vaith, H., Frenzel, R., Paschmann, G., and Melzner, F.: Electron gyro time measurement technique for determining electric and magnetic fields, in: Measurement Techniques in Space Plasmas: Fields, (Eds) Pfaff, R. F., Borovsky, J. E., and Young, D. T., 47-52, Geophysical Monograph 103, AGU, Washington, D. C., 1998. 